\title{
Composición Florística de la Finca Agroecológica de Zamorano, Honduras: Una Experiencia del Programa Aprender Haciendo
}

\section{Lilian Ferrufino-Acosta ${ }^{1}$, Rina Fabiola Díaz ${ }^{2}$, Denisse Benítez Nassar ${ }^{3}$, Flor de Rosa Atao ${ }^{4}$, Manuel García Chaclan ${ }^{5}$, Tania López ${ }^{6}$ y George E. Pilz}

Resumen. La finca agroecológica de Zamorano está en el valle de Yeguare, San Antonio de Oriente, Departamento de Francisco Morazán, Honduras, es comanejada por la Escuela Agrícola Panamericana, Zamorano. Esta finca tiene como propósito reproducir un sistema agroecológico que garantice su estructura, funcionamiento y biodiversidad. El objetivo de este estudio fue determinar su composición florística e identificar las especies de importancia ecológica y económica, así como su estado de conservación. Se identificaron 210 especies que pertenecen a 53 familias, las más comunes son Asteraceae, Fabaceae y Malvaceae. El 86\% de las especies registradas son nativas, en su mayoría hierbas y árboles de importancia económica. Las especies más importantes son: Guazuma ulmifolia, Quercus oleoides, Luehea candida, Bursera simaruba, Mimosa tenuiflora, Verbesina punctata, Malvaviscus arboreus. Solo ocho especies se encuentran en la categoría de atención menor, según la lista roja de la Unión Internacional para la Conservación de la Naturaleza (UICN), y dos especies de orquídeas en la lista CITES. La finca agroecológica de Zamorano ofrece un potencial económico por las especies de uso medicinal, maderable y comestible. Además, este estudio florístico se considera una herramienta para la elaboración del plan de manejo de la finca.

Palabras clave: Agroecología, diversidad especies, estructura ecológica, especies útiles, florística.

\section{Floristic composition in the Agroecological Farm of Zamorano, Honduras: An Experience of the Learning by Doing Program}

\begin{abstract}
The Zamorano agroecological farm is located in the valley of Yeguare and is managed by the Escuela Agrícola Panamericana, Zamorano. This farm aims to replicate an agroecological system that guarantees its structure, operation, and biodiversity. The objective of this study was to determine the floristic composition of the Zamorano agroecological farm and identify the species of ecological and economic importance, as well as the state of conservation of species. Two hundred and ten species belonging to 53 families were collected and identified, with the most common families being in the Asteraceae, Fabaceae and Malvaceae. Eighty-six percent of the registered species are native to Zamorano. In addition, very few introduced species were identified, mostly herbs and trees of economic importance. The most common species are: Guazuma ulmifolia Lam., Quercus oleoides Schltdl. \& Cham., Luehea candida Moç \& Sessé ex DC. Mart., Bursera simaruba (L.) Sarg., Mimosa tenuiflora (Willd.) Poir., Verbesina punctata Robins. \& Greenm, and Malvaviscus arboreus Cav.. Based on the list, only seven species are in the category of minor attention according to the red list of IUCN (The International Union for Conservation of Nature) and two species of

\footnotetext{
${ }^{1}$ Herbario Paul C. Standley (EAP), Carrera de Ambiente y Desarrollo, Escuela Agrícola Panamericana, Zamorano, Honduras; Herbario Cyril Hardy Nelson Sutherland (TEFH), Escuela de Biología, Universidad Nacional Autónoma de Honduras, Honduras, correo electrónico lilian.ferrufino@unah.edu.hn

${ }^{2}$ Herbario Paul C. Standley (EAP), Carrera de Ambiente y Desarrollo, Escuela Agrícola Panamericana, Zamorano, Honduras, correo electrónico rfdiaz@zamorano.edu

${ }^{3}$ Departamento de Recursos Naturales, Universidad del Estado de Kansas, Manhattan, Kansas, Estados Unidos, correo electrónico denissebn.94@hotmail.com

${ }^{4}$ Comunidad Paca, Anta, Cusco, Perú, correo electrónico florataohuillca11@gmail.com

${ }^{5}$ Carrera de Ambiente y Desarrollo, Escuela Agrícola Panamericana, Zamorano, Honduras, correo electrónico mjgarcia@zamorano.edu

${ }^{6}$ Comayagua, Comayagua, Honduras, correo electrónico tnlpz75@gmail.com

${ }^{7}$ Herbario Paul C. Standley (EAP), Carrera de Ambiente y Desarrollo, Escuela Agrícola Panamericana, Zamorano, Honduras, correo electrónico gpilz@zamorano.edu
} 
orchids in the list CITES (The Convention on International Trade in Endangered Species of Wild Fauna and Flora). The Zamorano agroecological farm offers economic potential with the registration of species for medicinal, timber and edible use. In addition, this floristic study is considered a tool for the elaboration of the management plan for the farm.

Key words: Agroecology, ecological structure, floristic, species diversity, useful species.

\section{Introducción}

Los bosques secos se distribuyen desde México hasta Argentina y las islas del Caribe, estos bosques son de los más amenazados y del que se conserva solo $10 \%$ en la mayoría de los países (Banda et al. 2016, Janzen 1988). Centroamérica y el norte de Sudamérica se clasifican dentro de los 12 grupos florísticos y cuentan con aproximadamente 808 especies y 121 sitios. Se considera que muy pocas especies se comparten entre los bosques secos del neotropico; sin embargo, especies como Guazuma ulmifolia se distribuyen en la mayoría de los sitios de bosque seco (Banda et al. 2016).

La mayor parte de los bosques secos de Honduras se encuentran en el sur, sureste y centro del país; estos están fragmentados debido a la creciente presión por las actividades antrópicas como la agricultura, la ganadería, la quema y extracción de madera que cada día amenazan las poblaciones de muchas especies en estos ecosistemas (Barrance et al. 2009). Las poblaciones humanas que viven en las zonas de bosques secos son generalmente de escasos recursos económicos por lo que para estas personas, el bosque es de gran importancia por los productos que este proporciona (madera, leña, forraje, frutas, medicina, casa y agua) (Barrance et al. 2009). Algunas de las especies arbóreas de importancia en estos ecosistemas son: Simarouba glauca, Cochlospermum vitifolium, Bursera simaruba, Tabebuia rosea, Guazuma ulmifolia, Cordia alliodora, Mimosa tenuiflora, Karwinskia calderonii, Cecropia peltata, Gliricidia sepium, Casearia corymbosa, Quercus oleoides y Luehea candida (Gillespie et al. 2000; Padilla 2003).

La flora de los bosques secos ha sido estudiada en varios países de Latinoamérica. Por ejemplo, los bosques secos del occidente de la provincia de Loja, en Ecuador, se consideran amenazados por la actividad antrópica que afecta el estado de conservación del ecosistema. En este contexto, se determinó la abundancia y distribución de las especies ecológicamente más importantes de esta zona, y se descubrió que Cavanillesia platanifolia y Tabebuia chrysantha son las más representativas (Vásquez et al. 2005). Un estudio de un inventario arbóreo se registra en el municipio de Guanarito, estado Portuguesa, en Venezuela; este se realizó en tres fincas para valorar los árboles forestales. Las especies más comunes fueron Chlorophora tinctorea, Guazuma ulmifolia y Samanea saman, en su mayoría son especies maderable (Solórzano et al. 2008).

En Honduras, también se ha estudiado la composición florística en bosques secos, principalmente en la región oriental. Gutiérrez et al., (2002) y Lesko-Madriz et al., (2005) trabajaron en el bosque ripario de Quebrada Grande y la selva baja caducifolia, así como en el bosque de galería de la Quebrada Güisisire. Menéndez y Melara (2002) realizaron estudios en Morocelí (Cardona Caravantes y Oramas Wenholz 2002). Estudios de la composición florística se han llevado a cabo en el cerro Masicarán en San Antonio de Oriente, Francisco Morazán, Honduras (Pérez Laniado 2004; Garcés López 2004; Illescas Burneo 2005) y en el Corredor Biológico de Uso Múltiple Texíguat (Carbajal 2010). Según Barrance et al. (2009), el sur de Honduras es una de las áreas de bosque seco en mesoamérica más alterada y considera que estos bosques albergan cerca de 85 especies de importancia económica en la industria de la madera, ornamental, artesanal y medicinal. Asimismo, es altamente probable que estos remanentes de bosque seco alberguen especies endémicas y amenazadas por actividades antrópicas.

La Escuela Agrícola Panamericana, Zamorano, firmó el 6 de diciembre del 2014 un convenio con la Agencia Suiza para el Desarrollo que establece un Centro Zamorano de Enseñanza en Agroecología (CZEA). El objetivo del centro es promover la producción en un agroecosistema que utiliza los recursos locales disponibles y conserva la 
biodiversidad local (Holguín Guerrero 2015). Como parte del CZEA, la finca agroecológica de Zamorano comienza a inicios del 2015 y está ubicada dentro de un bosque seco, por lo que el conocimiento de la flora existente en la finca es necesario para que los estudiantes y agricultores usen adecuadamente este recurso (Atao Huilca 2016; Benítez Nassar 2016).

El principio central de la agroecología es reproducir en la naturaleza un sistema productivo que debe repetir el funcionamiento del ecosistema natural, su ciclo de nutrientes, su estructura y a la vez aumentar la biodiversidad (Altieri 2002). La finca agroecológica de Zamorano provee a los alumnos un espacio para poner en práctica los fundamentos de la agroecología a través de un módulo de Aprender Haciendo impartido desde el primer trimestre del 2016 a estudiantes de segundo y cuarto año. Entre las actividades realizadas están: proyectos de tesis, diseños agroecológicos, análisis rápido de resiliencia, monitoreo de la flora y fauna, bioconstrucciones, cercas vivas, camas vivas, huacas, compostaje y laguna de cosecha de aguas.

El objetivo de este estudio fue determinar la composición florística (hierbas, arbustos y árboles) de las especies registradas en la finca agroecológica de Zamorano por los estudiantes de segundo y cuarto año de la clase 2016 y 2018 en el módulo de agroecología del aprender haciendo, considerando la estructura ecológica, importancia económica y el estado de conservación de las especies identificadas.

\section{Materiales y Métodos}

Descripción del área de estudio. La finca se ubica en el valle del Yeguare, municipio de San Antonio de Oriente, departamento de Francisco Morazán, a 33 $\mathrm{km}$ al sureste de la ciudad de Tegucigalpa, Honduras. La finca se localiza en $13^{\circ} 59^{\prime} 18.00^{\prime \prime} \mathrm{N}$, 8658'46.00" O, a 700-800 msnm (Figura 1).

La finca tiene 40.03 ha de un bosque seco tropical secundario cerca de la Escuela Agrícola Panamericana, Zamorano, Honduras. La cobertura vegetal está compuesta por bosque en regeneración, bosque de encino, pastizales, plantación de teca y bosque ripario. El suelo es franco-arenoso-arcilloso con un $\mathrm{pH}$ de 5.80 y un drenaje moderadamente lento (Cruz Ibarra y Pineda López, 2016).
Recolección de las muestras. Hubo giras de campo dos veces al mes en el módulo de agroecología del programa aprender haciendo durante el 2016 por los estudiantes de segundo y cuarto año de las clases 2016 y 2018, respectivamente. Se recolectaron muestras con flor y fruto de hierbas, trepadoras, arbustos, árboles y epífitas. Estas muestras fueron herborizadas (prensadas, secadas y etiquetadas) luego identificadas y depositadas en el Herbario Paul C. Standley (EAP). Algunas especies fueron identificadas por expertos botánicos con fotografías del material vegetal que fueron enviadas por correo electrónico (Figura 2).

Se establecieron 20 transectos de $100 \mathrm{~m}$ de largo y $2 \mathrm{~m}$ de ancho. Para comprender mejor la dinámica de la finca agroecológica de Zamorano, el estudio se complementó con el análisis de la estructura vertical en donde se clasificaron los árboles y arbustos en rangos de altura. Para analizar la estructura horizontal se midió el diámetro a la altura del pecho (DAP) estandarizada de $1.37 \mathrm{~m}$ y se agruparon por clases diamétricas. Se tomaron en cuenta arbustos con un DAP desde $0.1 \mathrm{~cm}$ para tener mayor representación de la vegetación. Algunos arbustos tenían crecimiento simpodial, en este caso, se midieron los tallos individuales y posteriormente se calculó un DAP total (Dt) según lo propuesto por Franco Rosselli et al. (1997) en la siguiente ecuación:

$$
\mathrm{Dt}=(4 \mathrm{At} / \pi) 1 / 2
$$

en donde: $A t=\Sigma A i$ y $A i=\pi(D A P)^{2} / 4$

Se midió la altura de los arbustos y árboles con un clinómetro (Suunto PM5/360PC). También se calculó el valor de importancia de especies (IVI) para arbustos y árboles basado en la suma de la abundancia, dominancia y frecuencia relativa de las especies registradas. Además, se visitaron los sitios oficiales en línea de la lista roja de la Unión Internacional para la Conservación de la Naturaleza (UICN) y la Convención sobre el Comercio Internacional de Especies Amenazadas de Fauna y Flora Silvestres (CITES) para revisar el estado de las poblaciones de cada especie e identificar si presentan alguna categoría de amenaza.

Usos potenciales. Las especies fueron agrupadas según su posible uso, de acuerdo a lo sugerido por Esquivel et al. (2003), en tres categorías: maderable, 
forrajera y frutal. Basado en la lista de especies identificadas se consultó en la literatura el uso potencial de las especies registradas en la finca (Berendsohn et al. 2009, 2012; Carrasquilla 2006; Castañeda 2004; Chízmar et al. 2009a, 2009b; De Sedas et al. 2010; House et al. 1995; Morales 2000; Toribio y Correa 2009).

\section{Resultados y Discusión}

Composición florística. Se registran 210 especies distribuidas en 53 familias de angiospermas (Cuadro 1). La mayoría de las especies son nativas (86\%) y $4 \%$ son especies introducidas (Figura 3 ). La mayoría de las especies introducidas tienen usos comestibles, Mangifera indica y Citrus reticulata son las más abundantes. Estas especies fueron introducidas en años previos por el propietario anterior de la finca. Entre las especies maderables introducidas, Tectona grandis, llamada comúnmente teca, fue la más abundante. Estudios en los bosques secos de Nicaragua y Costa Rica reportan número de especies y familias (204 especies y morfoespecies, 58 familias) muy cercanas a las encontradas en la finca agroecológica de Zamorano (Gillespie et al. 2000).

Las especies herbáceas son las más dominantes (69\%), seguidas de los árboles (22\%) y los arbustos $(9 \%)$. De las 53 familias registradas en la finca, las más diversas fueron: Asteraceae con 22 especies, en su mayoría herbáceas, seguida de Fabaceae con 18 especies y Malvaceae con 12 especies arbustivas (Figura 4). La finca presenta un bosque secundario; aquí se realizaron actividades agrícolas hace 10 años; sin embargo las plantas herbáceas son muy dominantes en la finca. No obstante, las leguminosas también predominan en estos bosques secos secundarios. Estudios en la región sugieren que Fabaceae es la familia de angiospermas más abundante en los bosques secos del Neotrópico (Gentry 1995, Gillespie et al. 2000).

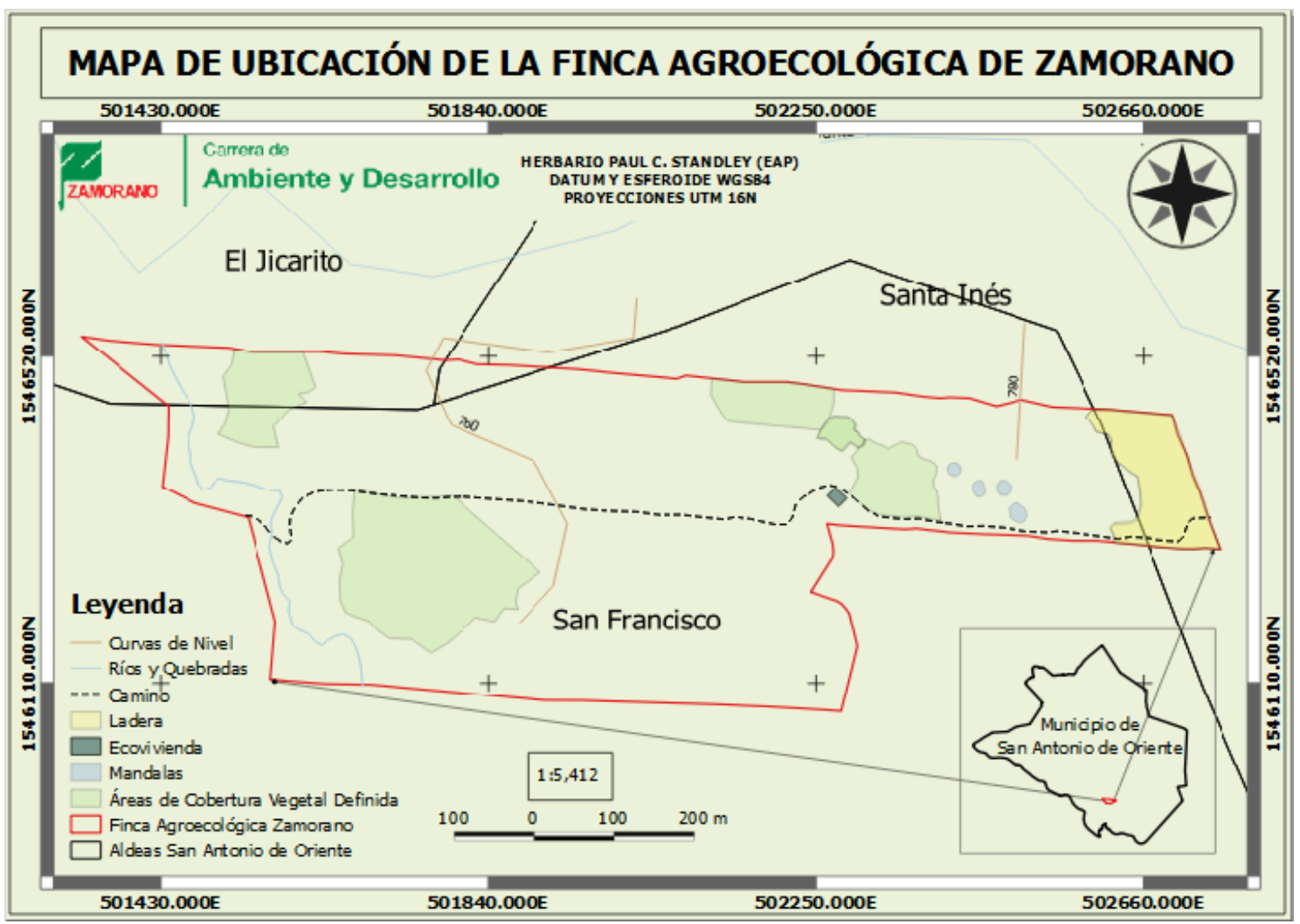

Figura 1. Ubicación de la finca agroecológica de Zamorano, Municipio de San Antonio de Oriente, Francisco Morazán, Honduras. 

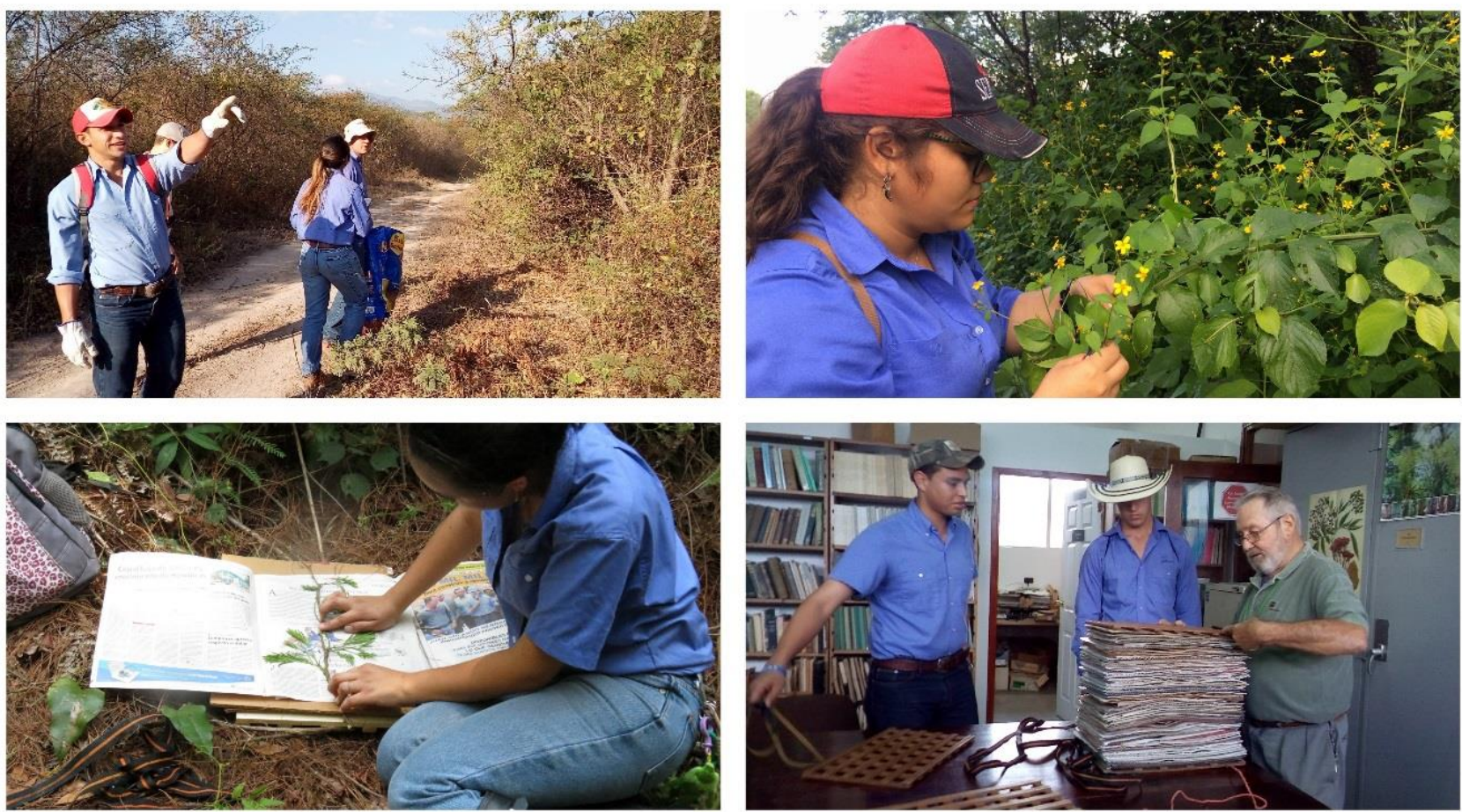

Figura 2. Recolecta y prensado del material botánico por los alumnos del módulo de Agroecología de segundo y cuarto año del Programa Aprender Haciendo, Escuela Agrícola Panamericana, Honduras.

Cuadro 1. Lista de las plantas vasculares registradas en la finca agroecológica de Zamorano colectadas durante el 2016. Los nombres comunes son consultados en Nelson (2008), House et al. (1995) y las etiquetas de los especímenes depositados en los herbarios Cyril Hardy Nelson Sutherland (TEFH) y Paul C. Standley (EAP). Hábito: H. hierba; A. árbol; Ar. arbusto. Origen: N. nativa; I. introducida.

\begin{tabular}{llll}
\hline Taxa & Hábito & Origen Nombres comunes \\
\hline
\end{tabular}

\section{ANGIOSPERMAS BASALES}

\begin{tabular}{llll} 
Aristolochiaceae & & & \\
\hline Aristolochia anguicida Jacq. & $\mathrm{H}$ & $\mathrm{N}$ & Algaria de monte, guaco \\
\hline Aristolochia maxima Jacq. & $\mathrm{H}$ & $\mathrm{N}$ & Guaco, guacolicor \\
\hline
\end{tabular}

\section{EUDICOTYLEDONEAE}

\begin{tabular}{llll} 
Acanthaceae & $\mathrm{H}$ & $\mathrm{N}$ & Coralillo, árbol de flecha \\
\hline Aphelandra scabra Juss. & $\mathrm{H}$ & $\mathrm{N}$ & Alambrito, camarón, yerba morada \\
\hline Blechum brownei (Lam.) Urb. & $\mathrm{H}$ & $\mathrm{N}$ & Hierba de cerdo \\
\hline Blechum pyramidatum Kunth & $\mathrm{H}$ & $\mathrm{N}$ & Cacagüillo, cola de caballo \\
\hline Elytraria imbricata Kunth & $\mathrm{H}$ & $\mathrm{I}$ & Gallina de guinea, pintada \\
\hline Hypoestes phyllostachya (Vahl) Pers. & $\mathrm{H}$ & $\mathrm{N}$ & Flor azul, flor de venado \\
\hline Ruellia geminiflora Baker & $\mathrm{H}$ & $\mathrm{N}$ & Cabro, pata de perico, zorrillo \\
\hline Ruellia inundata (Vahl) Sm. &
\end{tabular}


Cuadro 1. Continuación

\begin{tabular}{llll}
\hline Taxa & Hábito & Origen & Nombres comunes \\
\hline Amaranthaceae & & & \\
\hline Alternanthera brasiliana var. villosa (Moq.) Kuntze & $\mathrm{H}$ & $\mathrm{I}$ & Botoncito \\
\hline Alternanthera pubiflora (Benth.) Kuntze & $\mathrm{H}$ & $\mathrm{N}$ & Coyontura \\
\hline Gomphrena serrata L. & $\mathrm{H}$ & $\mathrm{N}$ & Botoncillo, sanguinaria, cabeza de pollo \\
\hline Iresine calea (Ibañez) Standl. & $\mathrm{H}$ & $\mathrm{N}$ & Cola de chivo, hierba de gato, mosquito \\
\hline Iresine diffusa Humb. \& Bonpl. ex Willd. & $\mathrm{H}$ & $\mathrm{N}$ & Coralillo, hierba de garo, pata de paloma \\
\hline Anacardiaceae & & & \\
\hline Mangifera indica L. & & & \\
\hline Spondias mombin L. & $\mathrm{A}$ & $\mathrm{N}$ & Mango \\
\hline Spondias purpurea L. & $\mathrm{A}$ & $\mathrm{N}$ & Jobo \\
\hline & $\mathrm{A}$ & $\mathrm{N}$ & Ciruela, jocote \\
\hline
\end{tabular}

\section{Apocynaceae}

\begin{tabular}{llll} 
Blepharodon mucronatum (Schltdl.) Decne. & $\mathrm{H}$ & $\mathrm{N}$ & Coyoles de venado, injerto \\
\hline Dictyanthus asper (Mill.) W.D. Stevens & $\mathrm{H}$ & $\mathrm{N}$ & Cachito, cuchinito \\
\hline Gonolobus barbatus Kunth & $\mathrm{H}$ & $\mathrm{N}$ & Champer, cuayote, matacoyote \\
\hline Macroscepis hirsuta (Vahl) Schltr. & $\mathrm{H}$ & $\mathrm{N}$ & Matabibi \\
\hline Mandevilla subsagittata (Ruiz \& Pav.) Woodson & $\mathrm{H}$ & $\mathrm{N}$ & Flor de mico \\
\hline Prestonia mexicana A. DC. & $\mathrm{H}$ & $\mathrm{N}$ & Cacho de venado lechoso \\
\hline Thevetia peruviana (Pers.) K. Schum. & $\mathrm{H}$ & $\mathrm{N}$ & Chilca, chilindrón \\
\hline
\end{tabular}

\section{Araliaceae}

\begin{tabular}{llll}
\hline Dendropanax arboreus Decne. \& Planch. & A & N & Concha de cangrejo, mano de león, mano de mico \\
\hline Oreopanax capitatus (Jacq.) Decne. \& Planch. & A & N & Mano de león, tambor \\
\hline
\end{tabular}

\begin{tabular}{|c|c|c|c|}
\hline Ageratum conyzoides L. & $\mathrm{H}$ & 1 & Hierba de pollo, flor azul, mejorana \\
\hline Ageratum corymbosum Zuccagni & $\mathrm{H}$ & $\mathrm{N}$ & Cielitos \\
\hline Baccharis trinervis Pers. & $\mathrm{H}$ & $\mathrm{N}$ & Lengua de vaca; cardenillo; alcotán \\
\hline Calea ternifolia Kunth & $\mathrm{H}$ & $\mathrm{N}$ & Amargoso, chirivito \\
\hline Chromolaena odorata (L.) R.M. King \& H. Rob. & $\mathrm{Ar}$ & $\mathrm{N}$ & Amargo, tres puntas, mejorana \\
\hline Cosmos sulphureus Cav. & $\mathrm{H}$ & $\mathrm{N}$ & Cambray, clavel de muerto \\
\hline Delilia berteroi Spreng. & $\mathrm{H}$ & $\mathrm{N}$ & Tostoncillo \\
\hline Elephantopus mollis Kunth & $\mathrm{H}$ & $\mathrm{N}$ & Alberrania, hoja de San Antonio, oreja de coyote \\
\hline Elephantopus spicatus Juss. ex Aubl. & $\mathrm{H}$ & $\mathrm{N}$ & Lechuguilla, escoba real \\
\hline Gnaphalium attenuatum $D C$. & $\mathrm{H}$ & $\mathrm{N}$ & Sanalatodo, gordolobo \\
\hline Melanthera nivea (L.) Small & $\mathrm{H}$ & $\mathrm{N}$ & Chichinguaste, flor de la vida, guácara \\
\hline Lasianthaea fruticosa (L.) K.M. Becker & $\mathrm{Ar}$ & $\mathrm{N}$ & Girasol, monte blanco, vara blanca \\
\hline Synedrella nodiflora (L.) Gaertn. & $\mathrm{H}$ & $\mathrm{N}$ & Cachito, cuernecillo, guácara \\
\hline Tagetes filifolia Lag. & $\mathrm{H}$ & $\mathrm{N}$ & Anisillo, anís de llano \\
\hline Tagetes lucida Cav. & $\mathrm{H}$ & 1 & Flor de muerto, pericón \\
\hline Tithonia tubiformis (Jacq.) Cass. & $\mathrm{Ar}$ & $\mathrm{N}$ & Crespillo, girasol, gigantón \\
\hline Tridax procumbens $L$. & $\mathrm{H}$ & $\mathrm{N}$ & Curagusano, girasol, hierba de toro \\
\hline Verbesina agricolarum Standl. \& Steyerm. & $\mathrm{Ar}$ & $\mathrm{N}$ & Chimaliote amarillo, flor de octubre, varilla negra \\
\hline Verbesina gigantea Jacq. & $\mathrm{Ar}$ & $\mathrm{N}$ & Abaco del monte, tabaquillo \\
\hline Verbesina guatemalensis B.L. Rob. \& Greenm. & $\mathrm{Ar}$ & $\mathrm{N}$ & Contraraña, vara de blanca, vara de San José \\
\hline Verbesina punctata B.L. Rob. \& Greenm. & $\mathrm{Ar}$ & $\mathrm{N}$ & Lengua de vaca \\
\hline Vernonia leiocarpa $D C$. & $\mathrm{Ar}$ & $\mathrm{N}$ & Quesillo, tatascán \\
\hline Vernonia patens Kunth & $\mathrm{Ar}$ & $\mathrm{N}$ & Apazotillo, barrehorno, tatascán \\
\hline Vernonia scorpioides (Lam.) Pers. & $\mathrm{Ar}$ & $\mathrm{N}$ & Añil, apazotillo, santo domingo \\
\hline Wamalchitamia williamsii (Standl. \& Steyerm.) Strother & & $\mathrm{N}$ & Tatascán \\
\hline
\end{tabular}


Ferrufino-Acosta et al. Composición Florística de la Finca Agroecológica de Zamorano, Honduras

Cuadro 1. Continuación

\begin{tabular}{llll}
\hline Taxa & Hábito & Origen & Nombres comunes \\
\hline Bignoniaceae & & & \\
\hline Amphilophium crucigerum (L.) L.G. Lohmann & $\mathrm{H}$ & $\mathrm{N}$ & Peine de mico \\
\hline Crescentia alata Kunth & $\mathrm{A}$ & $\mathrm{N}$ & Jícaro \\
\hline Handroanthus ochraceus (Cham.) Mattos & $\mathrm{A}$ & $\mathrm{N}$ & Cortés amarillo \\
\hline Tabebuia rosea (Bertol.) DC. & $\mathrm{A}$ & $\mathrm{N}$ & Macuelizo \\
\hline Bixaceae & & & \\
\hline Cochlospermum vitifolium (Willd.) Spreng. & $\mathrm{A}$ & $\mathrm{N}$ & Berberio, tecomazuche \\
\hline Boraginaceae & & & \\
\hline Cordia bullata (L.) Roem. \& Schult. & & $\mathrm{N}$ & Cabeza negra, moño de gato \\
\hline Cordia curassavica (Jacq.) Roem. \& Schult. & $\mathrm{Ar}$ & $\mathrm{N}$ & Carne asada, santo domingo, varilla negra \\
\hline Heliotropium curassavicum L. & $\mathrm{Ar}$ & $\mathrm{N}$ & Heliotropo pantanosos, cola de alacrán \\
\hline Burseraceae & $\mathrm{H}$ & & \\
\hline Bursera simaruba (L.) Sarg. & & & \\
\hline Cactaceae & $\mathrm{H}$ & $\mathrm{N}$ & Indio desnudo \\
\hline Acanthocereus tetragonus (L.) Hummelinck & & & \\
\hline Hylocereus undatus (Haw.) Britton et Rose & $\mathrm{Ar}$ & $\mathrm{N}$ & Pitahaya, saite, tuna cuadrada \\
\hline Opuntia lutea (Rose) D.R. Hunt & $\mathrm{Ar}$ & $\mathrm{N}$ & Hermosa noche, pitaya, pitaya roja \\
\hline
\end{tabular}

\section{Convolvulaceae}

Evolvulus alsinoides (L.) L. Ipomoea indica (Burm.) Merr.

Ipomoea minutiflora (M. Martens \& Galeotti) House

Ipomoea purpurea (L.) Roth

Ipomoea squamosa Choisy

Jacquemontia tamnifolia (L.) Griseb.

Turbina corymbosa (L.) Raf.

$\begin{array}{lll}\text { H } & \text { N } & \text { Pata de paloma, pate paloma } \\ \text { H } & \text { N } & \text { Campanilla } \\ \text { H } & \text { N } & \text { Bejuquillo } \\ \text { H } & \text { N } & \text { Campanilla, campanilla morada, batatilla } \\ \text { H } & \text { N } & \text { Campanilla, tripa de gallina } \\ \text { H } & \text { I } & \text { Ninguno } \\ \text { H } & \text { N } & \text { Campanilla, flor de pascua }\end{array}$

\section{Cucurbitaceae}

Melothria scabra Naudin

Momordica charantia L.

$\begin{array}{ll}\mathrm{H} & \mathrm{I} \\ \mathrm{H} & \mathrm{I}\end{array}$

Sandia de montaña, sandillita

Calaica, pepino de monte, calabacito

\section{Euphorbiaceae}

Acalypha arvensis Poepp.

Acalypha pseudalopecuroides $\mathrm{Pax}$

Chamaesyce hypericifolia (L.) Millsp.

Croton glandulosus L.

Croton heterochrous Baill.

Euphorbia heterophylla L.

\begin{tabular}{ll}
$\mathrm{H}$ & $\mathrm{N}$ \\
$\mathrm{H}$ & $\mathrm{N}$ \\
$\mathrm{H}$ & $\mathrm{N}$ \\
$\mathrm{H}$ & $\mathrm{N}$ \\
$\mathrm{H}$ & $\mathrm{N}$ \\
$\mathrm{H}$ & $\mathrm{N}$ \\
\hline
\end{tabular}

\begin{tabular}{ll} 
N & Cancerina, hierba del cáncer, olotillo \\
\hline N & Ninguno \\
\hline N & Hierba de la golondrina \\
N & Chacotote, chicote \\
N & Golondrina \\
\hline N & Golondrina, hierba de duende, pastor de monte \\
\hline
\end{tabular}

\section{Fabaceae}

\begin{tabular}{llll}
\hline Acacia farnesiana (L.) Willd. & A & N & Espino blanco, guarumo \\
\hline Calliandra grandifolia P.H. Allen & A & N & Cabello de ángel \\
\hline Centrosema pubescens Benth. & H & N & Frijolillo, gallinita, gallito \\
\hline Centrosema virginianum (L.) Benth. & $\mathrm{H}$ & $\mathrm{N}$ & Amarratabaco, frijolillo, gallinita, gallito \\
\hline Crotalaria retusa L. & $\mathrm{H}$ & $\mathrm{I}$ & Chinchín, chipilín, quiebraplatos \\
\hline Dalea cliffortiana Willd. & $\mathrm{H}$ & $\mathrm{N}$ & Azulillo, chaguitillo \\
\hline Desmodium triflorum (L.) DC. & $\mathrm{H}$ & $\mathrm{N}$ & Golondrina, oreja de ratón, platí \\
\hline Enterolobium cyclocarpum (Jacq.) Griseb. & $\mathrm{A}$ & $\mathrm{N}$ & Guanacaste \\
\hline
\end{tabular}


Cuadro 1. Continuación

\begin{tabular}{|c|c|c|c|}
\hline Taxa & Hábito & Origen & Nombres comunes \\
\hline Gliricidia sepium (Jacq.) Kunth ex Walp. & $\mathrm{A}$ & $\mathrm{N}$ & Cacagua, madreado \\
\hline \multicolumn{4}{|l|}{ Fabaceae } \\
\hline Indigofera hirsuta L. & $\mathrm{H}$ & 1 & Añil dulce, añil forrajero \\
\hline Inga laurina (Sw.) Willd. & A & $\mathrm{N}$ & Guama, másica \\
\hline Mimosa albida Humb. \& Bonpl. ex Willd. & $\mathrm{H}$ & $\mathrm{N}$ & Zarza, zarza de cerro, zarza hueca \\
\hline Mimosa pudica L. & $\mathrm{H}$ & $\mathrm{N}$ & Dormilona, zarza \\
\hline Mimosa tenuiflora (Willd.) Poir. & $\mathrm{A}, \mathrm{Ar}$ & $\mathrm{N}$ & Carbón \\
\hline Pachyrhizus erosus (L.) Urb. & $\mathrm{H}$ & $\mathrm{N}$ & Bejuco de frijolillo, frijol papa, jícama \\
\hline Stylosanthes guianensis (Aubl.) Sw. & $\mathrm{H}$ & $\mathrm{I}$ & Lengua de pájaro, alfabilla \\
\hline $\begin{array}{l}\text { Vigna adenantha (G. Mey.) Maréchal, Mascherpa \& } \\
\text { Stainier }\end{array}$ & $\mathrm{H}$ & $\mathrm{N}$ & Choreque, pollito \\
\hline Zornia reticulata $\mathrm{Sm}$. & $\mathrm{H}$ & $\mathrm{N}$ & Trencilla \\
\hline \multicolumn{4}{|l|}{ Fagaceae } \\
\hline Quercus oleoides Schltdl. \& Cham. & A & $\mathrm{N}$ & Encino, encino blanco, roble \\
\hline \multicolumn{4}{|l|}{ Gentianaceae } \\
\hline Schultesia brachyptera Cham. & $\mathrm{H}$ & $\mathrm{N}$ & Sulfatillo \\
\hline \multicolumn{4}{|l|}{ Hydroleaceae } \\
\hline Hydrolea spinosa L. & $\mathrm{H}$ & $\mathrm{N}$ & Bagre, espina de bagre, espina de chunte \\
\hline \multicolumn{4}{|l|}{ Lamiaceae } \\
\hline Hyptis brevipes Poit. & $\mathrm{H}$ & $\mathrm{N}$ & Botón de caballero \\
\hline Hyptis capitata Jacq. & $\mathrm{H}$ & $\mathrm{N}$ & Cabeza de pollo, miona, pan caliente \\
\hline $\begin{array}{l}\text { Mesosphaerum asperifolium (Standl.) Harley \& J.F.B. } \\
\text { Pastore }\end{array}$ & $\mathrm{Ar}$ & $\mathrm{N}$ & Ninguno \\
\hline Mesosphaerum urticoides (Kunth) Kuntze & $\mathrm{Ar}$ & $\mathrm{N}$ & Ninguno \\
\hline Salvia kellermanii Donn. Sm. & $\mathrm{H}$ & $\mathrm{N}$ & Chupamiel \\
\hline Salvia misella Kunth & $\mathrm{H}$ & 1 & Pegapega, mielillo, cola de alacrán \\
\hline Tectona grandis L. f. & A & 1 & Teca \\
\hline \multicolumn{4}{|l|}{ Lythraceae } \\
\hline Cuphea carthagenensis (Jacq.) J.F. Macbr. & $\mathrm{Ar}$ & $\mathrm{N}$ & Marta \\
\hline
\end{tabular}

\section{Malpighiaceae}

\begin{tabular}{llll}
\hline Byrsonima crassifolia (L.) Kunth & $\mathrm{A}$ & $\mathrm{N}$ & Nance, \\
\hline Gaudichaudia albida Schltdl. \& Cham & $\mathrm{H}$ & $\mathrm{N}$ & Ala de cucaracha, cantagallo, flor de novia \\
\hline Heteropterys brachiata (L.) DC. & $\mathrm{H}$ & $\mathrm{N}$ & Matapiojos \\
\hline Malvaceae & & & \\
\hline Ceiba pentandra (L.) Gaertn. & $\mathrm{A}$ & $\mathrm{N}$ & Ceiba \\
\hline Gaya calyptrata (Cav.) Kunth ex K. Schum. & $\mathrm{H}$ & $\mathrm{N}$ & Farolito \\
\hline Guazuma ulmifolia Lam. & $\mathrm{A}$ & $\mathrm{N}$ & Guácimo, caulote, tapaculo \\
\hline Heliocarpus donnellsmithii Rose & $\mathrm{A}$ & $\mathrm{N}$ & Damajao, majao, majao blanco \\
\hline Luehea candida (DC.) Mart. & $\mathrm{A}$ & $\mathrm{N}$ & Caulote blanco, guácimo blanco \\
\hline Malachra fasciata Jacq. & $\mathrm{H}$ & $\mathrm{N}$ & Malva brava \\
\hline Malvaviscus arboreus Cav. & $\mathrm{Ar}$ & $\mathrm{N}$ & Avispón, amapola, quesillo \\
\hline Malvaviscus penduliflorus DC. & $\mathrm{Ar}$ & $\mathrm{N}$ & Amapola, amapolita \\
\hline Sida acuta Burm. f. & $\mathrm{H}$ & $\mathrm{N}$ & Malva de macho, escobilla, escoba babosa \\
\hline Sida rhombifolia L. & $\mathrm{H}$ & $\mathrm{N}$ & Escoba, escobilla amarilla, malva \\
\hline Triumffeta calderonii Rose & $\mathrm{Ar}$ & $\mathrm{N}$ & Achiotillo, mozote de caballo \\
\hline
\end{tabular}


Ferrufino-Acosta et al. Composición Florística de la Finca Agroecológica de Zamorano, Honduras

Cuadro 1. Continuación

\begin{tabular}{|c|c|c|c|}
\hline Taxa & Hábito & Origen & Nombres comunes \\
\hline Waltheria indica L. & $\mathrm{H}$ & $\mathrm{N}$ & Escobilla, mazote de caballo, malva blanca \\
\hline \multicolumn{4}{|l|}{ Melastomataceae } \\
\hline Miconia albicans (Sw.) Steud. & $\mathrm{Ar}$ & $\mathrm{N}$ & Sirín negro, zarcil \\
\hline Tibouchina longifolia (Vahl) Baill. & $\mathrm{H}$ & $\mathrm{N}$ & Lengua de vaca, lengua de ciervo \\
\hline \multicolumn{4}{|l|}{ Menispermaceae } \\
\hline Cissampelos pareira L. & $\mathrm{H}$ & $\mathrm{N}$ & Alcotán, guaco, picamano \\
\hline \multicolumn{4}{|l|}{ Moraceae } \\
\hline Brosimum alicastrum Sw. & A & $\mathrm{N}$ & Másica, ojoche \\
\hline \multicolumn{4}{|l|}{ Myrtaceae } \\
\hline Acmena smithii (Poir.) Merr. \& L.M. Perry & A & 1 & Lilly Pilly \\
\hline Psidium guajava L. & A & $\mathrm{N}$ & Guayaba, guava \\
\hline Psidium guineense $\mathrm{Sw}$. & A & $\mathrm{I}$ & Guayaba agria, guayabilla \\
\hline Psidium sartorianum (O. Berg) Nied. & A & $\mathrm{N}$ & Guayabillo, arrayan \\
\hline Syzygium jambos (L.) Alston & A & $\mathrm{I}$ & Manzana rosa, manzana de castilla, pomarrosa \\
\hline \multicolumn{4}{|l|}{ Onagraceae } \\
\hline Ludwigia erecta (L.) H. Hara & $\mathrm{H}$ & $\mathrm{N}$ & Cigarrito, clavo, quilete \\
\hline Ludwigia octovalvis (Jacq.) P.H. Raven & $\mathrm{H}$ & $\mathrm{N}$ & Cigarrito, clavito, clavito de pozo \\
\hline \multicolumn{4}{|l|}{ Oxalidaceae } \\
\hline Oxalis neaei DC. & $\mathrm{H}$ & $\mathrm{N}$ & Destempla dientes \\
\hline \multicolumn{4}{|l|}{ Passifloraceae } \\
\hline Passiflora foetida $\mathrm{L}$. & $\mathrm{H}$ & $\mathrm{I}$ & Maracuyá silvestre \\
\hline Turnera ulmifolia L. & $\mathrm{H}$ & $\mathrm{N}$ & Escobón \\
\hline \multicolumn{4}{|l|}{ Phytolaccaceae } \\
\hline Phytolacca icosandra L. & $\mathrm{H}$ & $\mathrm{N}$ & Aguela, cola de ardilla, quilete \\
\hline \multicolumn{4}{|l|}{ Picramniaceae } \\
\hline Picramnia antidesma Sw. & A & $\mathrm{N}$ & Quina, corteza de Honduras \\
\hline \multicolumn{4}{|l|}{ Plantaginaceae } \\
\hline Scoparia dulcis L. & $\mathrm{H}$ & $\mathrm{N}$ & Escoba de castilla, arrocillo, culantro de pollo \\
\hline Russelia sarmentosa Jacq. & $\mathrm{H}$ & $\mathrm{N}$ & Coralillo, comida de culebra \\
\hline \multicolumn{4}{|l|}{ Polygalaceae } \\
\hline Asemeia violacea (Aubl.) J.F.B. Pastore \& J.R. Abbott & $\mathrm{H}$ & $\mathrm{N}$ & Ipecacuana, ipecuna, picacuana \\
\hline \multicolumn{4}{|l|}{ Portulacaceae } \\
\hline Portulaca oleracea L. & $\mathrm{H}$ & $\mathrm{I}$ & Verdolaga \\
\hline \multicolumn{4}{|l|}{ Primulaceae } \\
\hline Ardisia compressa Kunth & $\mathrm{Ar}$ & $\mathrm{N}$ & Capulín agrio \\
\hline \multicolumn{4}{|l|}{ Rhamnaceae } \\
\hline Clematis dioica $\mathrm{L}$. & $\mathrm{H}$ & $\mathrm{N}$ & Barba de viejo, crespillo \\
\hline Gouania lupuloides (L.) Urb. & $\mathrm{H}$ & $\mathrm{N}$ & Bejuco de mora, limpia dientes \\
\hline Karwinskia calderonii Standl. & A & $\mathrm{N}$ & Güiligüiste, pimientillo \\
\hline
\end{tabular}


Cuadro 1. Continuación

\begin{tabular}{llll}
\hline Taxa & Hábito & Origen & Nombres comunes \\
\hline Rubiaceae & & & \\
\hline Alibertia edulis (Rich.) A. Rich. ex DC. & $\mathrm{A}$ & $\mathrm{N}$ & Cachillo, cacho de venado, fruta de panilla \\
\hline Galium mexicanum Kunth & $\mathrm{H}$ & $\mathrm{N}$ & Hierba de la pulga \\
\hline Genipa americana L. & $\mathrm{A}$ & $\mathrm{N}$ & Jagua, irayol \\
\hline Guettarda deamii Standl. & $\mathrm{A}$ & $\mathrm{N}$ & Huesito, malacahuite \\
\hline Hamelia patens Jacq. & $\mathrm{Ar}$ & $\mathrm{N}$ & Canilla de venado, mazamorra, hojita dorada \\
\hline Rutaceae & & & \\
\hline Citrus aurantifolia Swingle & & & \\
\hline Citrus reticulata Blanco & $\mathrm{A}$ & $\mathrm{I}$ & Limón \\
\hline Fagara foliolosa (Donn. Sm.) Engl. & $\mathrm{A}$ & $\mathrm{I}$ & Mandarina \\
\hline Fagara riedeliana (Engl.) Engl. & $\mathrm{A}$ & $\mathrm{N}$ & Zarza rabo de iguana \\
\hline Murraya paniculata (L.) Jack & $\mathrm{A}$ & $\mathrm{N}$ & Cedrillo, Cedro espino, lagarto amarillo \\
\hline Salicaceae & $\mathrm{Ar}$ & $\mathrm{I}$ & Limonaria \\
\hline Casearia acuelata Jacq. & & & \\
\hline Casearia sylvestris Sw. & & & \\
\hline Prockia crucis P. Browne ex L. & $\mathrm{A}$ & $\mathrm{N}$ & Escabrón \\
\hline
\end{tabular}

\section{Santalaceae}

Phoradendron quadrangulare (Kunth) Griseb.

Phoradendron aguilarii Standl. \& Steyerm.

Phorandendron robustissimum Eichler

$\begin{array}{lll}\mathrm{Ar} & \mathrm{N} & \text { Matapalo } \\ \mathrm{Ar} & \mathrm{N} & \text { Ninguno } \\ \mathrm{Ar} & \mathrm{N} & \text { Muérdago, matapalo, suelda con suelda }\end{array}$

\section{Sapindaceae}

Allophylus camptostachys Radlk.

Cardiospermum corindum L.

Paullinia fuscescens Kunth.

Serjania grosii Schltdl.

Serjania triquetra Radlk.

$\begin{array}{cc}\mathrm{Ar} & \mathrm{N} \\ \mathrm{H} & \mathrm{N} \\ \mathrm{H} & \mathrm{N} \\ \mathrm{H} & \mathrm{N} \\ \mathrm{H} & \mathrm{I}\end{array}$

Nachillo, ratón

Bejuco de orinar, calzoncillo, farolitos

Bejuco de aparejo, campalca, chilmecate

Tronadora

Barbasco, bejuco tronador, bejuco de pescado

\section{Simaroubaceae}

Simarouba glauca DC.

A N Negrito, aceituno

\section{Solanaceae}

\begin{tabular}{llll}
\hline $\begin{array}{l}\text { Lycianthes arrazolensis (J.M. Coult. \& Donn. Sm.) } \\
\text { Bitter }\end{array}$ & $\mathrm{Ar}$ & $\mathrm{N}$ & Ninguno \\
\hline Solanum americanum Mill. & $\mathrm{H}$ & $\mathrm{N}$ & Hierbamora, güistomate \\
\hline Solanum capsicoides All. & $\mathrm{H}$ & $\mathrm{I}$ & Chichigua, tomatillo \\
\hline Solanum torvum Sw. & $\mathrm{Ar}$ & $\mathrm{N}$ & Friegaplatos, diente de perro \\
\hline Witheringia solanacea L'Hér. & $\mathrm{Ar}$ & $\mathrm{N}$ & Chile de montaña \\
\hline & & & \\
Urticaceae & $\mathrm{A}$ & $\mathrm{N}$ & Guarumo \\
\hline Cecropia peltata Vell. & & & \\
\hline & & & \\
Verbenaceae & $\mathrm{Ar}$ & $\mathrm{N}$ & Zopilote, zopilotillo \\
\hline Cornutia pyramidata L. & $\mathrm{H}$ & $\mathrm{N}$ & Cinco negritos \\
\hline Lantana camara L. & $\mathrm{Ar}$ & $\mathrm{N}$ & Juanilama, orégano de monte \\
\hline Lippia alba (Mill.) N.E. Br. ex Britton \& P. Wilson & $\mathrm{H}$ & $\mathrm{N}$ & Orégano, oreganito \\
\hline Lippia graveolens Kunth & $\mathrm{H}$ & $\mathrm{N}$ & Mozote, mozotillo, cola de alacrán \\
\hline Priva lappulacea (L.) Pers. & $\mathrm{H}$ & $\mathrm{N}$ & Rabito de alacrán, verbena \\
\hline Stachytarpheta angustifolia (Mill.) Vahl & &
\end{tabular}


Ferrufino-Acosta et al. Composición Florística de la Finca Agroecológica de Zamorano, Honduras

Cuadro 1. Continuación

\begin{tabular}{lccl}
\hline Taxa & Hábito & Origen & Nombres comunes \\
\hline Stachytarpheta jamaicensis (L.) Vahl & $\mathrm{H}$ & $\mathrm{N}$ & Verbena, golondrina \\
\hline Vitaceae & & & \\
\hline Vitis tiliifolia Humb. \& Bonpl. ex Schult. & $\mathrm{H}$ & $\mathrm{N}$ & Bejuco de agua, uva \\
\hline
\end{tabular}

\section{MONOCOTYLEDONEAE}

Alismataceae

\begin{tabular}{llll}
\hline Echinodorus grandiflorus (Cham. \& Schltdl.) Micheli & H & I & Ninguno
\end{tabular}

\section{Amaryllidaceae}

Crinum bulbispermum (Burm. f.) Milne-Redh. \&

Schweick.

Curculigo scorzonerifolia (Lam.) Baker

I Lirio

$\mathrm{H} \quad \mathrm{N} \quad$ Coquito

\section{Arecaceae}

\begin{tabular}{llll}
\hline Acrocomia mexicana Karw. ex Mart. & $\mathrm{H}$ & $\mathrm{N}$ & Coyol, olivo
\end{tabular}

\section{Bromeliaceae}

Bromelia plumieri (E. Morren) L.B. Sm.

Tillandsia caput-medusae E. Morren

Tillandsia fasciculata Sw.

Tillandsia paucifolia Baker

Tillandsia recurvata (L.) L.

Tillandsia schiedeana Steud.

$\mathrm{H} \quad \mathrm{N} \quad$ Motate, piñuela

$\mathrm{H} \quad \mathrm{N} \quad$ Bromelia

$\mathrm{H} \quad \mathrm{N} \quad$ Gallina ciega, gallito, gallinazo

$\mathrm{H} \quad \mathrm{N} \quad$ Ninguno

$\begin{array}{lll}\mathrm{H} & \mathrm{N} & \text { Gallito } \\ \mathrm{H} & \mathrm{N} & \text { Gallito, gallinazo }\end{array}$

\section{Commelinaceae}

Commelina diffusa Burm $f$

Commelina erecta $\mathrm{L}$.

$\mathrm{H} \quad \mathrm{N} \quad$ Hierba de pollo, tripa de pollo, siempreviva

$\mathrm{H} \quad \mathrm{I} \quad$ Verbena de agua, zacate de zorzal

\section{Cyperaceae}

Cyperus compressus L.

Cyperus esculentus L.

Cyperus virens Michx.

Eleocharis elegans (Kunth) Roem. \& Schult.

Rhynchospora nervosa (Vahl) Boeckeler

$\mathrm{H}$
$\mathrm{H}$
$\mathrm{H}$
$\mathrm{H}$
$\mathrm{H}$

\begin{tabular}{ll}
$\mathrm{N}$ & Coyolillo \\
\hline $\mathrm{I}$ & Coyolillo, tule \\
$\mathrm{N}$ & Coquillo \\
$\mathrm{N}$ & Junco, tule, tronadora \\
$\mathrm{N}$ & Cola de burro, coyolito, zacate estrella
\end{tabular}

\begin{tabular}{l} 
Orchidaceae \\
\hline Epidendrum verrucosum Sw. \\
\hline Guarianthe aurantiaca (Bateman ex Lindl.) Dressler \& \\
W.E. Higgins \\
\hline Trichocentrum cebolleta (Jacq.) M.W. Chase \& N.H. \\
Williams
\end{tabular}

$\begin{array}{lll}\mathrm{H} & \mathrm{N} & \text { Lilia de pascua } \\ \mathrm{H} & \mathrm{N} & \text { Lirio de palo } \\ \mathrm{H} & \mathrm{N} & \text { Lluvia de oro }\end{array}$

\section{Poaceae}

Ixophorus unisetus (J. Presl) Schltdl.

Lasiacis ruscifolia (Kunth) Hitchc.

Oplismenus burmannii (Retz.) P. Beauv.

Panicum maximum Jacq.

Phyllostachys aurea Carrière ex Rivière \& C. Rivière

Setaria tenax (Rich.) Desv.

Setaria vulpiseta (Lam.) Roem. \& Schult.

$\begin{array}{lcl}\text { H } & \text { I } & \text { Pasto blanco, pasto de leche, zacate de Honduras } \\ \text { H } & \text { N } & \text { Bera, caña dulce, carrizo } \\ \text { H } & \text { N } & \text { Zacate conejo, zacate gusano } \\ \text { H } & \text { I } & \text { Pasto guinea, zacate de la india, zacatón } \\ \text { H } & \text { I } & \text { Bambú dorado } \\ \text { H } & \text { N } & \text { Cola de zorro } \\ \text { H } & \text { N } & \text { Pasto blanco, zacate blanco }\end{array}$


Cuadro 1. Continuación

\begin{tabular}{llll}
\hline Taxa & Hábito & Origen & Nombres comunes \\
\hline MONILOPHYTA & & & \\
& & \\
\hline Thelypteridaceae & & & \\
\hline Thelypteris dentata (Forssk.) E.P. St. John & $\mathrm{H}$ & $\mathrm{N}$ & Rabo de gato \\
\hline
\end{tabular}
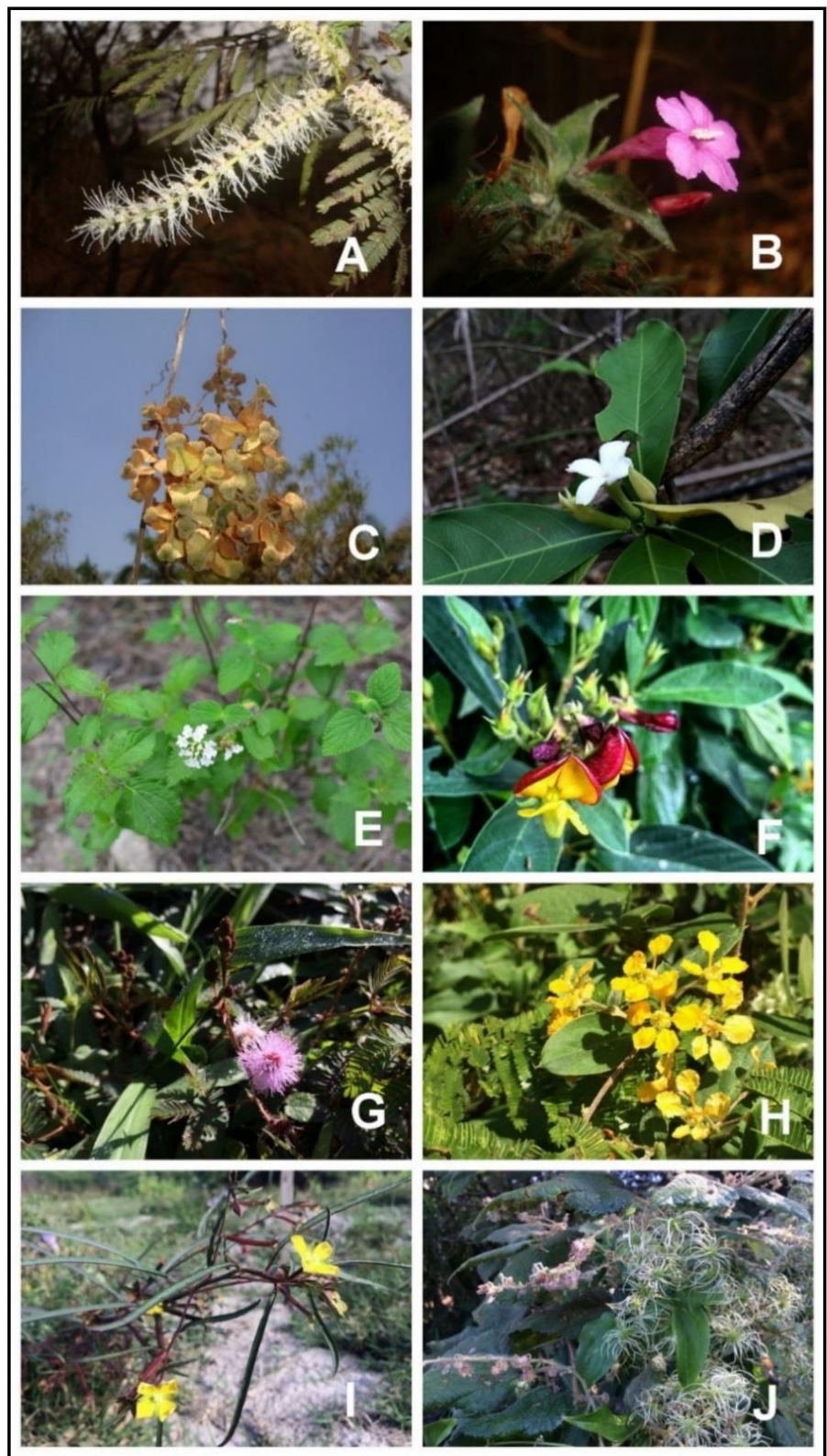

Figura 3. Especies de plantas registradas en la finca agroecológica de Zamorano, Honduras. A. Mimosa tenuiflora, B. Ruellia inundata, C. Serjania triquetra, D. Genipa americana, E. Lippia alba, F. Crotalaria retusa, G. Mimosa pudica, H. Heteropterys brachiata, I. Ludwigia octovalvis, J. Clematis dioica. 


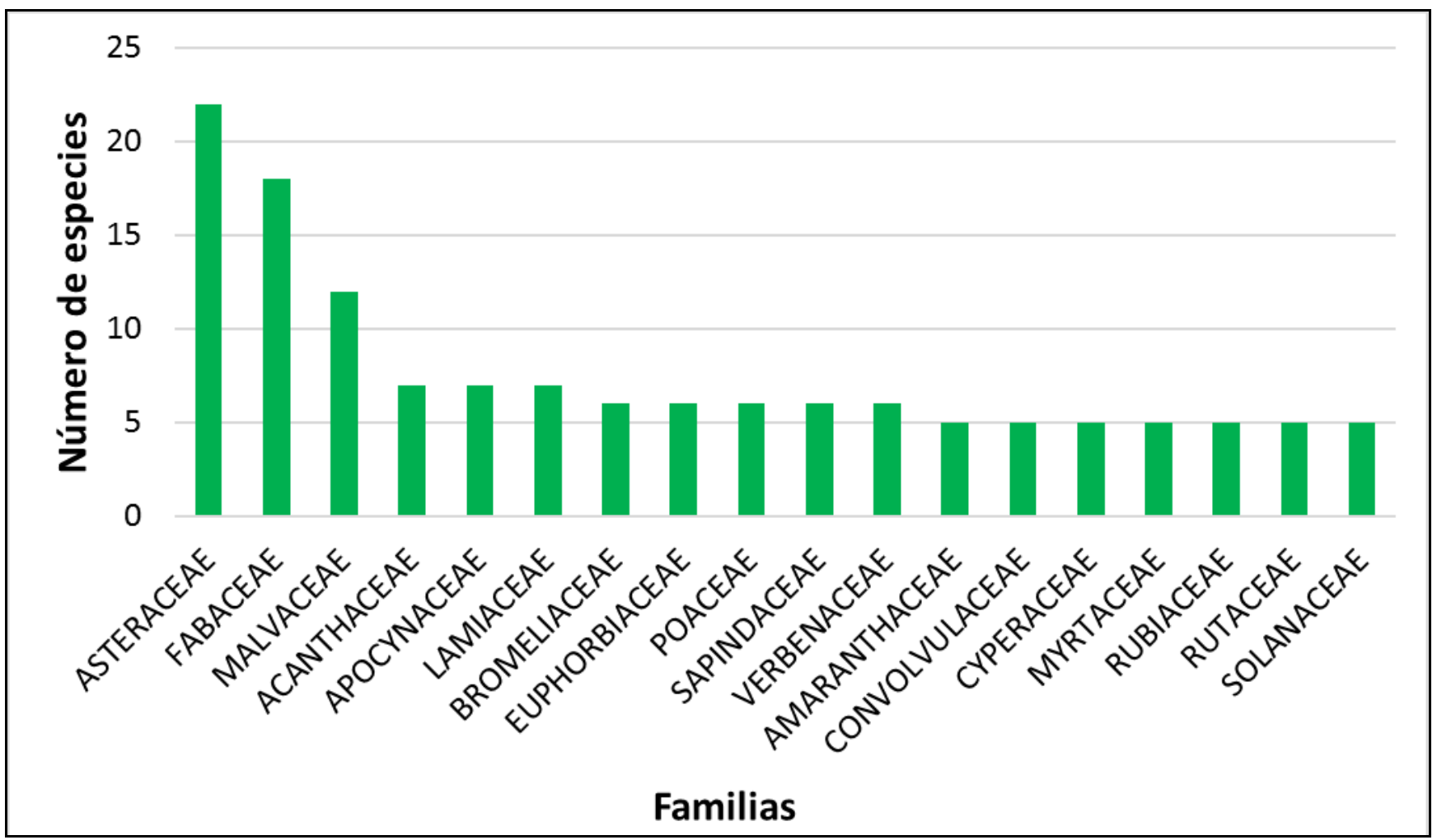

Figura 4. Número de especies por las 18 familias más diversas en la finca agroecológica de Zamorano, Honduras.

Estructura de la vegetación. Basado en los resultados del estudio de Benítez Nassar (2016) se registran entre las especies arbóreas más importantes Guazuma ulmifolia, Quercus oleiodes, Luehea candida y Bursera simaruba con un IVI de $48.07 \%, 33.98 \%, 30.94 \%$ y 19.64 , respectivamente. Atao Huillca (2016) reporta las especies arbustivas más importantes: Mimosa tenuiflora, Verbesina punctata, Malvaviscus arboreus y Verbesina gigantea con un IVI de 49.24, 28.50, 21.41 y $21.16 \%$, respectivamente. En el bosque seco de Masicarán, Valle de Yeguare, San Antonio de Oriente, un fragmento de bosque cerca de $2.5 \mathrm{~km}$ de la finca, registra entre sus especies más abundantes a Heliocarpus tormentosus, seguida por $Q$. oleoides y M. tenuiflora (Illescas Burneo 2005).

La estructura de la vegetación hace referencia a la distribución, organización y asociación de las plantas en un área determinada (Acosta et al. 2006). El estudio de estas características proporciona información sobre la dinámica de una región permitiendo diseñar medidas de aprovechamiento, manejo y conservación adecuadas (Alvis 2009).

Referente a la estratificación vertical, la máxima altura registrada fue de $18 \mathrm{~m}$, no obstante poco más del $6 \%$ de los individuos muestreados presentaron alturas medias a altas (10.1-20.0 m) ya que la mayor parte de los árboles y arbustos están en los rangos más bajos (Figura 5). La altura media para los arboles fue de $6 \mathrm{~m}$ y el $84 \%$ de los individuos muestreados presentaron alturas entre 1-10 m (Figura 5A). Se registran muy pocos individuos mayores a $10 \mathrm{~m}$, esto coincide con la decisión de dejar árboles dispersos en el terreno para sombra del ganado, así como un bosque ripario para la protección de zonas productoras de agua como la quebrada de la finca. Se observa que el $70 \%$ de arbustos muestreados no superan los $2 \mathrm{~m}$ (Figura $5 B$ ), esto evidencia el estado juvenil de este bosque.

Los análisis por clases diamétricas revelan la capacidad regeneradora de un bosque tomando en 
cuenta la permanencia de las especies dentro de una comunidad (Ajbilou et al. 2003; Moret et al. 2008). El $84 \%$ de los árboles tienen un DAP de $1.0-10.0 \mathrm{~cm}$ (Figura 6A). La segunda categoría diamétrica (10.1$20.0 \mathrm{~cm}$ ) es solamente $15 \%$ del total de los árboles. Entre los arbustos, el mayor número de individuos ocurrió entre las dos primeras categorías diamétricas (0.1-1.0 $\mathrm{m}$ y $1.1-2.0 \mathrm{~m}$ ) que representan el $73 \%$ de los arbustos

(Figura $6 \mathrm{~B})$.
En los arbustos, la especiemás representada entre 0.1 a $2.0 \mathrm{~cm}$ de DAP fue Vernonia punctata con 41 individuos que suponen el $16 \%$ del total, le siguen Malvaviscus arboreus con $11 \%$, Verbesina gigantea con $10 \%$ y Ageratum corymbosum el $9 \%$ dentro de este rango. No obstante, la tercera categoría diamétrica $(2.1-3.0 \mathrm{~cm})$ también estuvo bien representada con el $26 \%$ de los arbustos analizados.

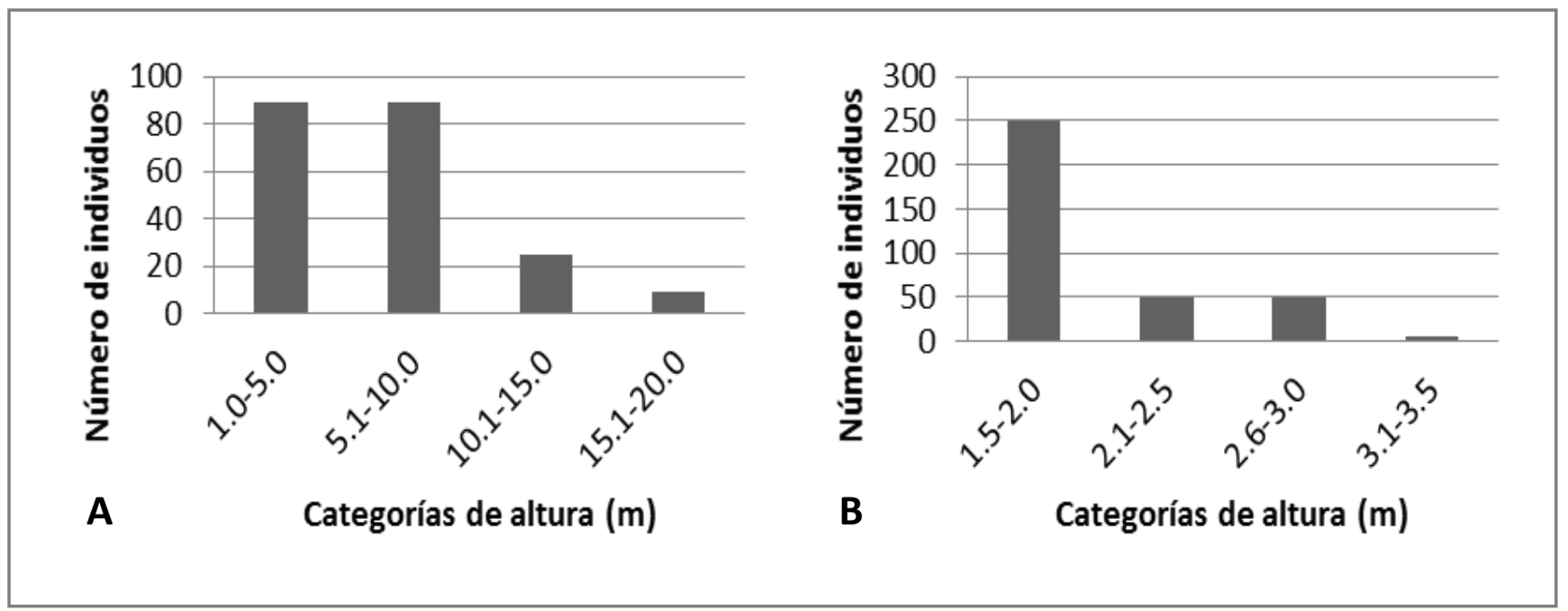

Figura 5. Estratificación vertical de los componentes: A. arbóreo y B. arbustivo de la finca agroecológica de Zamorano, Honduras.

Un estudio de Zarco et al. 2010 tomó en cuenta diámetros a partir de $1.0 \mathrm{~cm}$ obteniendo resultado similares. Ellos coinciden que los bosques poco intervenidos presentan categorías diamétricas inferiores. Esta tendencia indica que su permanencia y estabilidad están aseguradas. Del mismo modo, otros autores relatan que este comportamiento garantiza la recuperación de un bosque ya que presentan buena reproducción, establecimiento y regeneración natural continuos (Villa 2006; Godínez y López 2002).

Hubo una relación directa entre la estructura vertical y el análisis por clases diamétricas. Ambas estructuras presentan la misma forma de $\mathrm{J}$ invertida. El bosque tiene una alta densidad de árboles en los estratos más bajos. Esta estructura indica que es un bosque juvenil en estado de regeneración (Cascante Marín y Estrada Chavarría 2012).
Usos potenciales de la finca. La diversidad vegetal de la finca agroecológica de Zamorano incluye plantas con diferentes usos, activos y potenciales, entre los que destaca el uso medicinal. El $30 \%$ de las especies tienen este tipo de uso, un dato relevante tomando en cuenta que la finca se encuentra ubicada entre comunidades que pueden aprender a explotar este recurso debido al auge de la fitoterapia durante los últimos años (Cañigueral et al. 2003).

Las especies medicinales más conocidas son Blechum browneii (camarón), Acrocomia mexicana (coyol), Amphilophium crucigerum (peine de mico), Heliocarpus donellsmithii (majagua), Cissampelos pareira (hoja de terciopelo), Passiflora foetida (maracuyá silvestre), Lantana camara (cinco negritos), Stachytarpheta angustifolia (verbena), Lippia alba (juanilama u orégano de monte), Vitis tiliifolia (uvita), Gomphrena serrata (botoncillo), 
Ferrufino-Acosta et al. Composición Florística de la Finca Agroecológica de Zamorano, Honduras

Phytolacca icosandra y Calliandra grandifolia (cabello de ángel) (Muñoz y Pitty 1994; Pitty y Molina 1998). Esta variedad de especies usadas en la medicina tradicional constituye un semillero para estudios etnobotánicos y fitoquímicos (Beyra et al. 2004).

Considerando el servicio a largo plazo que podría proporcionar la finca, hay un potencial maderable de unas especies. Este terreno muestra un proceso de sucesión joven, representado por hierbas altas y arbustos de bajo porte, son los bosques secundarios quienes suplen la demanda de madera en varias regiones del neotrópico (Wadsworth 1997). Las especies maderables en la finca son Thevetia peruviana (campana amarilla), Dendropanax arboreus (mano de león), Inga laurina (guama), Cochlospermum vitifolium (berberio), Mimosa tenuiflora (carbón), Quercus oleoides (encino blanco), Tectona grandis (teca), Ceiba pentandra (ceiba), Brosimum alicastrum (másica), Guazuma ulmifolia (guazimo), Casearia aculeata (escabrón), Cecropia peltata (guarumo) y Bursera simaruba (indio desnudo) (Berendsohn et al. 2009, Carrasquilla 2006; Castañeda 2004; Chízmar et al. 2009a, 2009b; De Sedas et al. 2010; House et al. 1995; Morales 2000; Toribio y Correa 2009). Algunas de estas especies también fueron registradas por Aguirre (2012).
Las especies maderables también son usadas como cercas vivas y proporcionan ventajas ecológicas y económicas. Las plantas usadas como cercas vivas pueden crecer naturalmente 0 propagarse con mucha facilidad por estacas (Villanueva et al. 2008). Estas cercas tienen larga duración y son especies resistentes que además generan productos de valor económico como leña, forraje y frutos, por ejemplo Mimosa tenuiflora y Acacia farnesiana. Se suman a estos setos vivos las cactáceas Acanthocereus tetragonus (saite), Hylocereus undatus (pitaya) y Opuntia lutea (nopal, tuna). Estos cactus también producen frutos comestibles y en México sus tallos son consumidos como verduras (Cerén López et al. 2015).

Tabebuia rosea (macuelizo), Handroanthus ochraceus (cortés amarillo) y Gliricidia sepium (madreado), además de ser maderables y medicinales, son ornamentales por la vistosidad de sus flores. Byrsonima crassifolia (nance), Mangifera indica (mango), Spondias mombin (jobo), Spondias purpurea (ciruela), Crescentia alata (jícaro), Syzygium jambos (manzana rosa) y Psidium guajava (guayaba) son especies importantes en la seguridad alimentaria de la región (Berendsohn et al. 2009, Chízmar 2009a, De Sedas et al. 2010) (Figura 7).

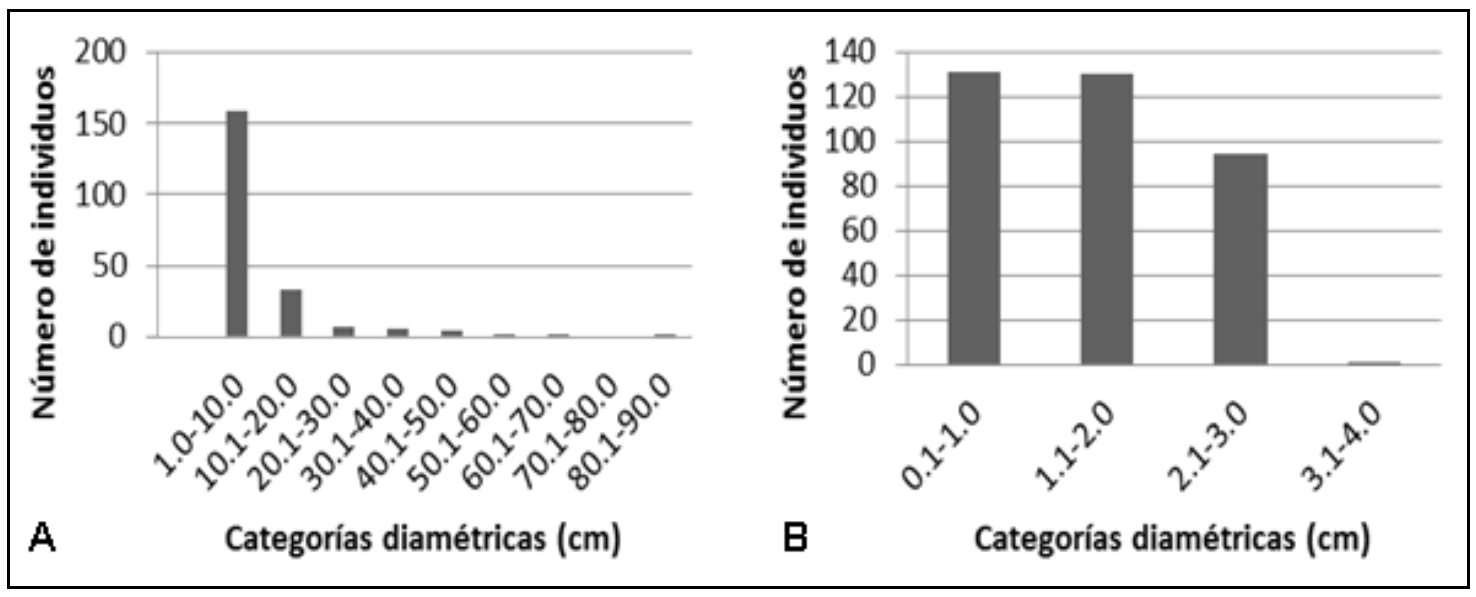

Figura 6. Distribución diamétrica: A. arbóreo y B. arbustivo de la finca agroecológica de Zamorano, Honduras. 


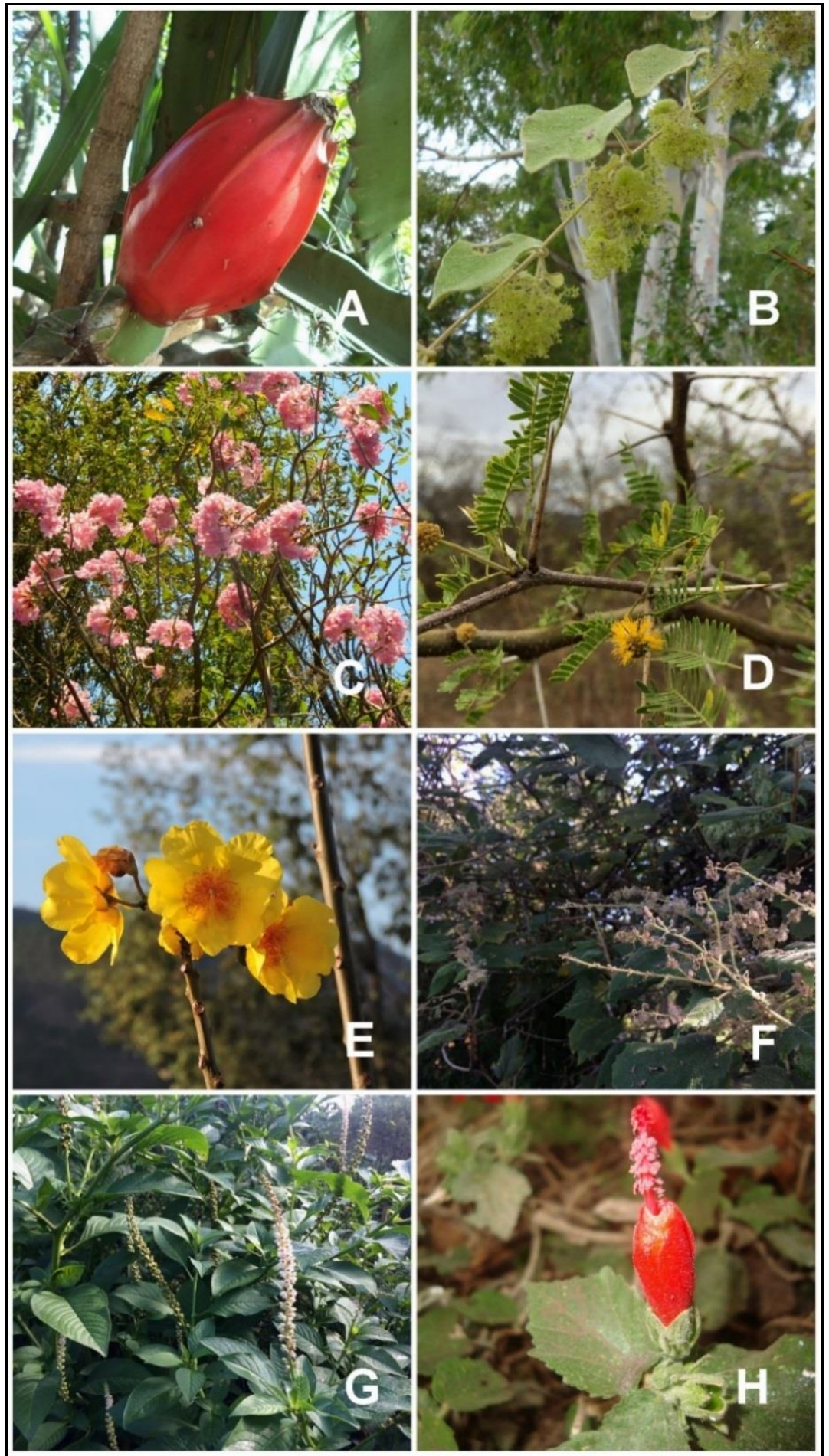

Figura 7. Especies con importancia económica de la finca agroecológica de Zamorano, Honduras. A. Acanthocereus tetragonus, B. Cissampelos pareira, C. Tabebuia rosea, D. Acacia farnesiana, E. Cochlospermum vitifolium, F. Triumfetta calderonii, G. Phytolacca icosandra, H. Malvaviscus arboreus. 
Algunos árboles, como la ceiba y el macuelizo, son usados en sistemas agropastoriles para sombra del ganado. Los arbustos de la finca juegan un papel fundamental en la conservación del suelo, entre ellos, Simarouba glauca (negrito, aceituno), Casearia sylvestris (sombra de armado), Psidium guineense (guayabo agrio), Indigofera hirsuta (añil dulce) y Pachyrhizus erosus (jícama), evitan la erosión, funcionan como abono verde, restauran suelos dañados e interactúan con bacterias fijadoras de nitrógeno para mejorar la fertilidad del suelo (Portillo et al. 2009). Además, el $81 \%$ de estas especies son nativas, lo que confiere a la finca un ecosistema equilibrado y permite que estas plantas puedan utilizarse en programas de reforestación local (Vásquez Yánez et al. s.f.).

Barrance et al. (2009) enlistan los árboles económicamente más importantes en las fincas del sur de Honduras y comentan que las plantas en su mayoría son usadas para leña, construcción y comida (frutos). No obstante, en la finca agroecológica la mayoría de las especies, en particular las herbáceas, tienen usos medicinales. Estos resultados concuerdan con los obtenidos por Albuquerque et al. (2005), quienes comentan que la mayoría de las especies registradas en un bosque seco secundario y disturbado en el noroeste de Brasil, son plantas herbáceas de uso medicinal.

Estado de conservación. De las 210 especies identificadas en la finca, ocho están en la lista roja de UICN con atención menor: Mimosa albida, Mimosa pudica, Heliotropium curassavicum, Cyperus compressus, Tillandsia fasciculata, Casearia aculeata, Commelina diffusa, Bromelia plumieri, Gaya calyptrata y Acanthocereus tetragonus. En el apéndice II de CITES están Trichocentrum cebolleta y Guarianthe aurantiaca (Orchidaceae).

En la finca agroecológica hay pocas especies con prioridad en su conservación. Barrance et al. (2009) comentan que en los agrosistemas de los bosques secos tropicales se ha dado poca prioridad al estado de conservación global; pero esto facilita el desarrollo de estrategias para el manejo y uso de la diversidad de especies arbóreas en estos ecosistemas. Se destaca la importancia de las fincas para la conservación de polinizadores que juegan un rol muy importante en la seguridad alimentaria, en particular las abejas nativas que han sufrido un declive en los últimos años (United States Department of Agriculture et al. 2008).

\section{Conclusiones}

En la finca agroecologíca Zamorano habitan al menos 210 especies. La mayoría de las especies registradas son nativas y algunas introducidas, y la mayoría son plantas herbáceas.

La altura media de los árboles y arbusto es de 3 $\mathrm{m}$, y la mayoría está entre 1-5 m, muy pocos se encuentran sobre los $10 \mathrm{~m}$. La mayor parte de estas especies tiene un diámetro a la altura del pecho de 1$10 \mathrm{~cm}$. Es decir, la estructura vertical y horizontal presenta una $\mathrm{J}$ invertida. Esta estructura se debe a que las poblaciones presentan un estado juvenil y en proceso de regeneración de un bosque secundario.

Las especies arbóreas más abundantes fueron: Luehea candida, Tectona grandis, Guazuma ulmifolia, Guettarda deamii y Quercus oleoides. Las especies arbustivas son: Mimosa tenuiflora, Verbesina punctata, Malvaviscus arboreus, Verbesina gigantea, Cordia bullata y Heliocarpus donnellsmithii. No cabe duda que conocer el estado de conservación de las especies que se registran ocho especies en la finca según la lista roja de UICN y dos especies en el apéndice II en la lista CITES.

La finca provee bienes ecosistémicos, en particular las especies herbáceas que tienen propiedades medicinales. La mayoría de las especies arbóreas son usadas para madera, leña, comestible, pasto para ganado y algunas con importancia medicinal. Basado en estas especies de importancia económica y ecológica, se pueden establecer sistemas silvícolas y de agroforestería.

El aprender haciendo, con la incorporación de los estudiantes del módulo de agroecología, permitió que el monitoreo se extendiera todo un año para obtener un muestreo representativo de la finca agroecológica de Zamorano. Además, es un punto de partida para que a través de los programas estudiantiles, Zamorano reconozca las prioridades de las comunidades aledañas y prepare estrategias de manejo de especies con prioridad de uso, así como el desarrollo integral. 


\section{Agradecimientos}

Los autores agradecen a los estudiantes del módulo de agroecología de segundo y cuarto año del Programa Aprender haciendo de la Escuela Agrícola Panamericana, Zamorano, de la clase 2016 y 2018, así como los botánicos, Douglas Stevens (MO), Leslie Landrum (ASU), Dagoberto Rodríguez (LAGU), German Sandoval y Olvin Oyuela (TEFH) que colaboraron en la identificación del material vegetal.

\section{Literatura Citada}

Acosta, V., P. Araujo y M. Iturre. 2006. Caracteres estructurales de las masas. Facultad de Ciencias Forestales, Universidad Nacional de Santiago de Estero, Santiago de Estero, Argentina. Serie Didáctica No 22.

Aguirre, Z. 2012. Especies forestales de los bosques secos del Ecuador. Ministerio del Ambiente de Ecuador-MAE y la Organización de las Naciones Unidas para la Agricultura y la Alimentación-FAO. Quito, Ecuador. 140p.

Ajbilou, R., T. Marañón y J. Arroyo. 2003. Distribución de clases diamétricas y conservación de bosques en el norte de Marruecos. Investigación agraria. Sistemas y recursos forestales 12(2): 111-123.

Albuquerque, U.P., L.H.C. Andrade y A.C.O. Silva. 2005. Use of plant resources in a seasonal dry forest (Northeastern Brazil). Acta Botanica Brasilica 19(1): 2738.

Alvis, J.F. 2009. Análisis estructural de un bosque natural localizado en zona rural del municipio de Popayán. Facultad de Ciencias Agropecuarias 7(1): 115-122.

Altieri, M.A. 2002. Agroecology: the science of natural resource management. Agriculture, Ecosystems \& Environment 93 (1-3):1-24.

Atao Huillca, F.R. 2016. Composición florística de arbustos de la finca agroecológica de Zamorano, Valle del Yeguare, Honduras. Tesis Ing. Ambiente y Desarrollo, Zamorano, Honduras. 22 p.

Barrance, A., K. Schreckenberg y J. Gordon. 2009. Conservación mediante el uso: Lecciones aprendidas en el bosque seco tropical mesoamericano. Londres, UK. Overseas Development Institute. 141p.

Benítez Nassar, D.M. 2016. Composición florística de árboles de la finca agroecológica de Zamorano, Valle El Yeguare, Honduras. Tesis Ing. Ambiente y Desarrollo, Zamorano, Honduras. 26 p.

Berendsohn, W.G., A.K. Gruber y J.A. Monterrosa Salomón. 2009. Nova silva cuscatlanica. Árboles nativos e introducidos de El Salvador. Parte 1: Angiospermae - Familias A a L. Englera 29(1): 1-438.
Berendsohn, W.G., A.K. Gruber, J.A. Monterrosa Salomón y G.M. Molina. 2012. Nova Silva Cuscatlanica. Árboles nativos e introducidos de El Salvador. Parte 2: Angiospermae - Familias M a $\mathrm{P}$ y Pteridophyta. Englera 29(2): 1-300.

Beyra, A., M. León, E. Iglesias, D. Ferrándiz, R. Herrera, G. Volpato, D. Godínez, M. Guimarais y R. Álvarez. 2004. Estudios etnobotánicos sobre plantas medicinales en la provincia de Camagüey (Cuba). Anales del Jardín Botánico de Madrid 61(2): 185-204

Cañigueral, S., E. Dellacassa y A. Bandoni. 2003. Plantas Medicinales y Fitoterapia: ¿Indicadores de dependencia - factores de desarrollo? Acta Farmacéutica Bonaerense 22 (3): 265-78.

Carbajal Vásquez, J.S. 2010. Vegetación del "Corredor Biológico de Uso Múltiple Texiguat”, El Paraíso, Honduras. Tesis Ing. Desarrollo Socioeconómico y Ambiente, Zamorano, Honduras. 53 p.

Cascante Marín, A. y A. Estrada Chavarría. 2012. Diversidad y composición del fragmento más importante de bosque premontano del Valle Central de Costa Rica. Brenesia 77:57-70.

Cardona Caravantes, R.A. y S. A. Oramas Wenholz. 2002. Composición florística de la selva baja caducifolia en Quebrada Grande, Morocelí, El Paraíso. Tesis Ing. Desarrollo Socioeconómico y Ambiente, Zamorano, Honduras. $26 \mathrm{p}$.

Carrasquilla, L. 2006. Árboles y arbustos de Panamá. Editora Novo Art, S.A. Panamá. 478 p.

Castañeda, C. 2004. Árboles y arbustos de los bosques secos de Guatemala. Instituto Nacional de Bosques (INAB). Guatemala. 199 p.

Cerén López, J.G., J.E. Menjívar Cruz y A.S. Arias Montes. 2015. Diversidad de cactus de El Salvador. Museo de Historia Natural de El Salvador. 152 p.

Chizmar F.C., A.M. Correa, V.G. Chang, C.S. Lobo, H.A. Quesada, L.J.G. Cerén, L.R. Lara, C.J.E. Menjívar, V.I. Ruíz, P.R. House, M.T. Mejía y G.I Coronado. 2009a. Plantas comestibles de Centroamérica. Primera edición. Instituto Nacional de Biodiversidad, INBio. Santo Domingo de Heredia, Costa Rica. 360 p.

Chízmar, F.C., A. Lu y M. Correa. 2009b. Plantas de uso folclórico y tradicional en Panamá. Instituto Nacional de Biodiversidad (INBio). Santo Domingo de Heredia, Costa Rica. 132 p.

CITES. 2017. Convención sobre el Comercio Internacional de Especies Amenazadas de Fauna y Flora Silvestres. Recuperado en enero 2017, de https://cites.org.

Cruz Ibarra, M.A. y C.M. Pineda López. 2016. Calidad de los suelos de los Llanos de Ocotal y Finca Agroecológica y actualización de los índices de calidad de los suelos de "La L", Portón y Caoba. Zamorano, Honduras. Tesis Ing. Agr., Zamorano, Honduras. 24 p. 
De Sedas, A., F. Hernández, R. Carranza, M. Correa y M. Stapf. 2010. Guía de árboles y arbustos del campus Dr. Octavio Méndez Pereira. Universidad de Panamá. Instituto Nacional de Biodiversidad, INBio. Santo Domingo de Heredia, Costa Rica. $161 \mathrm{p}$.

Duery Salek, S. 2001. Caracterización del bosque seco de la comunidad de Oropolí, Honduras. Tesis Ing. Agr., Zamorano, Honduras. $78 \mathrm{p}$.

Esquivel, H., M. Ibrahim, C.A. Harvey, C. Villanueva, T. Benjamin y F.L. Sinclair. 2003. Árboles dispersos en potreros de fincas ganaderas en un ecosistema seco en Costa Rica. Agroforestería en las Américas 10 (3940): 24-29.

Franco Rosselli, P., J. Betancur y J.L. Fernández Alonso. 1997. Diversidad florística en dos bosques subandinos del sur de Colombia. Caldasia 19(1-2): 205-234

Garcés López, P.Á. 2004. Composición florística del bosque seco "Masicarán", Valle del Yeguare, Honduras, C.A. Tesis Ing. Desarrollo Socioeconómico y Ambiente, Zamorano, Honduras. $21 \mathrm{p}$.

Gentry, A.H. 1995. Diversity and floristic composition of neotropical dry forests. En S.H. Bullock, H.A. Mooney y E. Medina, Seasonally dry tropical forests (146-194). New York, Cambridge, USA: Press Syndicate of the University of Cambridge.

Gillespie, T.W., A. Grijalva y C.N. Farris. 2000. Diversity, composition, and structure of tropical dry forest in Central America. Plant Ecology 147 (1): 37-47.

Godínez, O. y L. López. 2002. Estructura, composición, riqueza y diversidad de árboles en tres muestras de selva mediana subperennifolia. Anales del Instituto de Biología, Universidad Nacional Autónoma de México, Serie Botánica 73(2): 283-314.

Holguín Guerrero, J.M. 2015. Aplicación de proceso de diseño ecológico al módulo de manejo integrado de cultivos y cambio climático. Tesis Ing. Ambiente y Desarrollo, Zamorano, Honduras. 50 p.

House, P.R., S. Lagos, L. Ochoa, C. Torres, T. Mejía y M. Rivas. 1995. Plantas medicinales comunes de Honduras. Sin editorial. Tegucigalpa, M.D.C., Honduras, $455 \mathrm{p}$.

Illescas Burneo, W. 2005. Caracterización florística del bosque seco Masicarán, Tatumbla, Honduras. C.A. Tesis Ing. Agr., Zamorano, Honduras. 78 p.

Janzen, D.H. 1988. Tropical dry forests the most endangered tropical ecosystem. En Biodiversity, E.O. Wilson (ed). National Academy Press, USA. p. 130137.

Morales, J. 2000. Bromelias de Costa Rica. Instituto Nacional de Biodiversidad (INBio). Santo Domingo de Heredia, Costa Rica. 182 p.Moret, A., L. Valera, A.
Mora, V. Garay, M. Jerez, M. Plonczak, M. Ramírez y D. Hernández. 2008. Estructura horizontal y vertical de Pachira quinata (Jacq.) W.S. Alverson (Bombacaceae) en el bosque universitario "El Caimital", Barinas, Venezuela. Ecotrópicos 21(2):62-74.

Muñoz, R. y A. Pitty. 1994. Guía Fotográfica para la identificación de Malezas, Parte I. Zamorano Academic Press, Zamorano, Honduras. 124 p.

Padilla, E.G. 2003. Estado de la diversidad biológica de los árboles y bosques de Honduras. Documentos de Trabajo: Recursos Genéticos Forestales. En Taller Regional sobre los Recursos Genéticos Forestales de Centroamérica, Cuba y México, Turrialba, Costa Rica.

Pérez, E., B. Richers, F. DeClerck, F. Casanoves, J. Gobbi y T. Benjamin. 2011. Uso y manejo de la cobertura arbórea en sistemas silvopastoriles en la subcuenca del Río Copán, Honduras. Agroforestería en las Américas. 16 (48): 26-35.

Pérez Laniado, M.E. 2004. Composición florística del Cerro Masicarán, San Antonio de Oriente, Honduras. Tesis Ing. Desarrollo Socioeconómico y Ambiente, Zamorano, Honduras. 44 p.

Pitty, A. y A. Molina R. 1998. Guía Fotográfica para la Identificación de Malezas: Parte II. Zamorano Academic Press, Zamorano, Honduras. 136 p.

Portillo, A.B., R.C. Razz García, M. Marin y O. Araujo Febres. 2009. Dinámica de crecimiento en plantas de añil dulce (Indigofera hirsuta L.). Asociación Latinoamericana de Producción Animal 17(3 y 4): 9196.

Siles, P., F.A. Rugama y L. Molina. 2013. Diversidad arbórea en cercas vivas y dos fragmentos de bosque en la comunidad de Santa Adelaida, Estelí, Nicaragua. Encuentro 96: 60-76.

Solórzano, N., M. Escalona, C. Zambrano, N. Aranda, J. Molina y L. Blanco. 2008. Inventario de árboles en potreros en fincas del municipio Guanarito, estado Portuguesa. Revista Unellez Ciencia y Tecnología 24: 8-16.

Toribio, N. y M. Correa. 2009. Semillas y frutos de uso artesanal en Panamá. Instituto Nacional de Biodiversidad (INBio). Santo Domingo de Heredia, Costa Rica. 108 p.

TROPICOS.org Missouri Botanical Garden. Recuperado en diciembre 2016, de http://www.tropicos.org/

UICN. The IUCN Red List of Threatened Species. Recuperado en diciembre 2016, de http://www.iucnredlist.org.

United States Department of Agriculture (USDA), Natural Resources Conservation Service (NRCS), Xerces Society for Invertebrate Conservation, \& San Francisco State University. 2008. Using Farm Bill Programs for Pollinator Conservation. Technical Note No. 78, 12 p. 
Vázquez, M.A., J.F. Freire y L. Suárez (eds.). 2005. Biodiversidad en los bosques secos de la zona de Cerro Negro-Cazaderos, occidente de la provincia de Loja: un reporte de las evaluaciones ecológicas y socioeconómicas rápidas. EcoCiencia, MAE y Proyecto Bosque Seco. Quito, Ecuador. 127 p.

Vásquez Yánez, C., A.I. Batis Muñoz, M.I. Alcocer Silva, M. Gual Díaz y C. Sánchez Dirso. S.f. Árboles y arbustos nativos potencialmente valiosos para la restauración ecológica y la reforestación. Instituto de Ecología, Universidad Nacional Autónoma de México. Comisión Nacional para el Conocimiento y Uso de la Biodiversidad (CONABIO) PROYECTO J-084. México, C.M. 15 p.

Villa, A. 2006. Caracterización diamétrica de las especies maderables en bosques primarios del Cerro Murrucucú. Gestión y Ambiente 9(2): 73-90.
Villanueva, C., I. Muhammad y F. Casasola. 2008. Valor económico y ecológico de las cercas vivas en fincas y paisajes ganaderos. Centro Agronómico Tropical de Investigación y Enseñanza (CATIE). Serie técnica. Informe técnico/CATIE; no. 372. Turrialba, Costa Rica. 36 p.Wadsworth, F.H. 1997. Producción Forestal para América Tropical. U.S. Dept. of Agriculture, Forest Service. Agriculture Handbook no. 710. Washington, DC. 563 p.

Zarco, V.M., J.I. Valdéz, G. Ángeles y O. Castillo. 2010. Estructura y diversidad de la vegetación arbórea del Parque Estatal Agua Blanca, Macuspana, Tabasco. Universidad y Ciencia, Trópico Húmedo 26(1): 1-17.

Recibido para publicación el 19 de diciembre del 2016. Aceptado para publicación el 26 de julio del 2017. Publicado el 12 de febrero del 2018. 